\title{
VALIDATION OF AN ELISA KIT FOR DETECTION OF ANTIBODIES AGAINST ASF VIRUS IN BLOOD OR SPLEEN OF DOMESTIC PIGS AND WILD BOARS
}

\author{
O.M. STRIZHAKOVA, V.M. LYSKA, A.S. MALOGOLOVKIN, M.B. NOVIKOVA, \\ M.V. SIDLIK, I.V. NOGINA, A.E. SHKAEV, E.A. BALASHOVA, V.V. KURINNOV,
} A.P. VASIL'EV

All-Russian Institute of Veterinary Virology and Microbiology, Federal Agency of Scientific Organizations, 1, ul. Akademika Bakuleva, pos. Vol'ginskii, Petushinskii Region, Vladimir Province, 601120 Russia, e-mail OMStr@yandex.ru Received September 25, 2016

\section{Abstract}

The causal agent of African swine fever (ASF) is a DNA virus belonging to Asfaviridae family which affects both wild boar Sus scrofa and domestic pig Sus scrofa domestica. Special features of the course of (ASF and its forms should be considered for the effective use of various ASF diagnostic methods aimed at the pathogen or specific antibody identification. ASF diagnosis in the wild boar is of special importance. The wild boar susceptibility to ASF virus is well known, and the disease has been repeatedly reproduced in experiments and detected in European wild boars in natural conditions. It is not unfrequently that when shooting wild boars, only organ samples are delivered to laboratories, so we decided to estimate the diagnostic value of the antibodies detected in tissues and evaluate an earlier developed test system for its efficacy when used for the assay. This report represents the results of validation of an indirect ELISA (a commercial kit «VNIIVViM ASF-ELISA $\mathrm{Ab} / \mathrm{Ag}$ ) $\mathrm{ASF}$ virus specific antibody detection in blood serum and spleen tissue extracts. For comparison, an indirect immunofluorescence assay (indirect IFA) was used. To estimate the obtained results, ROC analysis was applied. Examination of positive $(n=66)$ and negative $(n=410)$ porcine blood serum samples using indirect ELISA showed high sensitivity and specificity of the method with reference to IFA. Among the 476 serum samples examined in indirect ELISA, only 8 sera (1.6\%) were within the positive/negative cutoff area. The highest sensitivity (100\%) and specificity $(99.27 \%)$ for indirect ELISA when examining both domestic pig and wild boar blood sera were determined at a cutoff value of 0.264 . The samples of spleen extracts to be used for the study were collected from clinically healthy wild boars in ASF-affected Smolensk region in 2013 to 2014. In view of an acute form of the disease, we can suspect that the animals exhibiting positive reaction were in a latency period of the pathogeny. When examining the positive $(n=59)$ and negative $(n=678)$ spleen extracts, we also determined high sensitivity and specificity levels of indirect ELISA with reference to indirect IFA. Among the samples examined $(n=737), 10(1.3 \%)$ samples of spleen extracts were within the positive/negative cutoff area. The highest sensitivity $(100 \%)$ and specificity $(98.82 \%)$ were observed at a cutoff value of 0.284 . Thus, we confirmed the assay to be effective for porcine blood sera with $100 \%$ sensitivity (94.6 to $100 \%$ ) and $99.27 \%$ specificity $(97.90$ to $99.80 \%$ ) and for spleen extracts with $100 \%$ sensitivity ( 93.90 to $100 \%$ ) and $98.82 \%$ specificity ( 97.70 to $99.50 \%$ ).

Keywords: African swine fever, specific antibody, indirect ELISA, ROC analysis

African swine fever (ASF) is a highly contagious disease with $100 \%$ mortality when acute course, leading to significant economic losses [1]. ASF is characterized by fever, hemorrhagic diathesis and necro-degenerative changes in parenchymal organs [2-5]. The causative agent of ASF is a DNA-containing virus of Asfarviridae family [6]. There are hyperacute, acute, subacute, chronic, and latent ASF [7-9]. Special features of the ASF course and its forms should be considered for the effective use of various ASF diagnostic methods [10] aimed at the identification of the pathogen or specific antibodies. If hyperacute and acute forms, detection of virus-specific antibodies is possible only in spleen samples, as specific antibody producing cells and, respectively, the very antibodies appear there on day 2 to 3 post-infection, whereas the animals die on days 3-7 [11, 12]. 
In subacute and chronic disease, virus-specific antibodies are found in blood on days 7-10 [13-16], so earlier a detection of the viral antigen or genome in blood is advisable [17], since viremia is typical of this ASF period [17-19].

ASF diagnosis in the wild boar is of special importance as their susceptibility to ASF virus (ASFV) is well known. The disease has been repeatedly reproduced in experiments and detected in European wild boars under natural conditions [20].

In the Russian Federation, ASFV has been circulating in the populations of domestic pigs (Sus scrofa domestica) and wild boars (Sus scrofa) since November 2007 until present. High virulence of ASFV, circulating in the Russian Federation [21], and the acute ASF proven indicates basic importance of direct virus detection by PCR and direct immunofluorescence test (DIFT) in the scheme of the laboratory diagnosis [15). At the same time, long-time persistence of ASFV in wild boar populations in the territory of Russia suggests the possibility of endemic infection, so serological survey could be carried out more widely, especially in the disease-affected areas of the Russian Federation. When shooting wild boars, only organ samples are often delivered to laboratories, so we decided to estimate the diagnostic value of the antibodies detected in tissues and to validate for this a VNIIVViM ASF-ELISA Ab/Ag kit. Earlier, we used random samples of spleen extracts from dead ASFV-infected domestic pigs and wild boars when studying diagnostic value of specific tissue antibodies [16]. Indirect immunofluorescence test (IIFT) detects tissue anti-ASFV antibodies in spleen extracts of $49 \%$ domestic pigs and $33 \%$ wild boars (PCR- and DIFT-positive) died of acute ASF. IIFT is considered a reference test for the detection of antiASFV antibodies [22], but, along with high sensitivity and specificity, it has a low performance and some technological inconveniences. These necessitate the development, validation and practical use of ELISA methods which make it possible to detect specific antibodies not only in blood, but also in tissue extracts of infected animals.

In recent years, the ROC (receiver operating characteristic) analysis [2325 ] is often used in medicine and veterinary science, which allows, in the presence of positive and negative reference samples, to determine cut-off points, both numerically and graphically, and validate protocols by true positive rate/false positive rate of the objects classified as possessing a trait. When processing data using ROC curves, it is necessary to determine the cut-off level. Without this value it is impossible to perform calculations and interpret the positive and negative results [26, 27].

We have performed validation of a VNIIVViM ASF-ELISA Ab/Ag kit (AllRussian Research Institute of Veterinary Virology and Microbiology, VNIIVViM) for the detection of ASFV specific antibodies both in the blood serum and the tissue using ELISA test and ROC-analysis.

The purpose of the study was to estimate the operational characteristics of the developed commercial kit for indirect immunoassay (ELISA), VNIIVViM ASF-ELISA Ab/Ag, versus indirect immunofluorescence assay (IIFT) when detecting specific anti-ASFV antibodies in blood and tissue extracts of infected animals and to find an optimum cutoff point providing high reliability of the test.

Technique. Reference positive samples were collected randomly and independently from domestic pigs and wild boars in the experiment or in ASF outbreaks. The reference positive samples included 43 blood sera from 10 pigs experimentally infected with avirulent ASFV strain PSA-1-NH $\left(10^{-4.5} \mathrm{TCD}_{50} / \mathrm{ml}\right)$ collected during infection development, and 23 sera from slaughtered domestic pigs and wild boars shot at ASF outbreaks. For tissue extracts, 59 spleen samples were collected from clinically healthy wild boars shot in 2013-2014 in ASF- 
affected Smolensk region (the presence of specific antibodies was confirmed in IIFT). Absence of anti-ASFV antibodies in all reference negative samples was confirmed by IIFT (the reference test recommended by the World Organization for Animal Health, OIE, France), PCR and DIFT to be sure of no viral infection. The reference negative samples included 410 blood sera of domestic pigs and shot wild boars from historically ASF-free Russian farms and territories, and 678 PCR-, DIFT- and IIFT-negative spleen extracts from shot wild boars (ASFaffected Smolensk region, 2013-2014).

Blood sera were centrifuged at $2000 \mathrm{rpm}$. The samples for ELISA were diluted (1:50) with Tris- $\mathrm{HCl}$ buffer containing casein $(0.01 \mathrm{M}$ Tris- $\mathrm{HCl}, 0.154 \mathrm{M}$ $\mathrm{NaCl}, 0.5 \%$ casein, $\mathrm{pH} 7.8$ ), as recommended in the VNIIVViM ASF-ELISA $\mathrm{Ab} / \mathrm{Ag}$ kit instructions.

For tissue extracts, the spleen samples $(1 \mathrm{~g})$ were homogenized in a sterile $0.85 \% \mathrm{NaCl}\left(5 \mathrm{~cm}^{3}\right)$. The resulting $20 \%$ homogenates (weight/volume) were frozen twice at $-18 \pm 2{ }^{\circ} \mathrm{C}$, thawn at room temperature, and subjected to centrifugation (3000 rpm, 10-15 min). The supernatant (extract) was used in indirect IIFT and indirect ELISA (without additional dilution) to detect tissue-specific anti-ASFV antibodies

IIFT was performed with a commercial Kit for differential immunofluorescence diagnostics of African swine fever, classical swine fever, Aujeszky's disease (VNIIVViM). For test, we used African green monkey kidney cell culture CV-1 (Collection of VNIIVViM cell cultures) infected with avirulent nonhemesorbing ASFV strain 691/88 (State VNIIVViM Collection of Microorganisms). a fluorescence microscope Eclipse E200 (Nikon Corp., Japan) was used for luminescent microscopy with documentation.

A commercial VNIIVViM ASF-ELISA Ab/Ag kit for ELISA diagnostics was used in accordance to the recommended protocol. The results interpretation was based on a standard deviation principle (2.5-fold difference). For the MedCalc program, $\mathrm{S} / \mathrm{P}$, the ratio of optical density in the test sample to the positive control, was calculated using OD at $\lambda=405 \mathrm{~nm}$ (spectrophotometer Sunrise ${ }^{\mathrm{TM}}$, Tecan Austria GmbH, Austria) as follows:

$$
\frac{\mathrm{S}}{\mathrm{P}}=\frac{\mathrm{OD}_{\text {mean }} \text { test sample }-\mathrm{OD}_{\text {mean }} \text { negative control }}{\mathrm{OD}_{\text {mean }} \text { positive control }-\mathrm{OD}_{\text {mean }} \text { negative control }},
$$

ROC curves for assessment of the cutoff point and statistical analysis were constructed by $\mathrm{S} / \mathrm{P}$ values. The $\mathrm{S} / \mathrm{P}$ positive and negative samples in IIFT served as Diagnosis 1 and Diagnosis 0, respectively. The data were entered in the MedCalc program according to the procedure for the current version (available at http://www.medcalc.org).

1. ELISA and IIFT detection of anti-ASFV antibodies in blood of experimentally infected pigs and healthy animals

\begin{tabular}{l|c|c|c}
\hline \multirow{2}{*}{ ELISA } & \multicolumn{3}{|c}{ IIFT } \\
\cline { 2 - 4 } & positive & negative & total \\
\hline Positive & 43 & 2 & 45 \\
Negative & 0 & 98 & 98 \\
Total & 43 & 100 & 143 \\
\hline N o t e. Commercial diagnostic kits (All-Russian Research Insti- \\
tute of Veterinary Virology and Microbiology) were used.
\end{tabular}

Results. Relative sensitivity and relative specificity of indirect ELISA (VNIIVViM ASFELISA $\mathrm{Ab} / \mathrm{Ag}$ kit) were determined vs. IIFT ("gold standard" test). When analyzing blood sera (Table 1) from experimentally infected domestic pigs $(n=43)$ and healthy animals (historically ASF-free farms, $n=100$ ), the relative sensitivity was $100 \%$ (43 samples out of 143 were positive both in ELISA and IIFT, and 2 samples were positive in ELISA only), relative specificity was $98 \%$ at $95 \%$ confidence interval.

When checking the value of positive/negative cutoff for indirect ELISA based on the results of testing 66 positive and 410 negative serum samples from do- 
mestic pigs and wild boars, a diagram was plotted (Fig. 1) for the frequency of distribution of the values obtained. A total of 5 reference positive and 19 reference negative samples were co-located in the S/P interval from 0.2 to 0.4 , that is the optical densities of the samples within this range could be classified both as false negative and false positive.

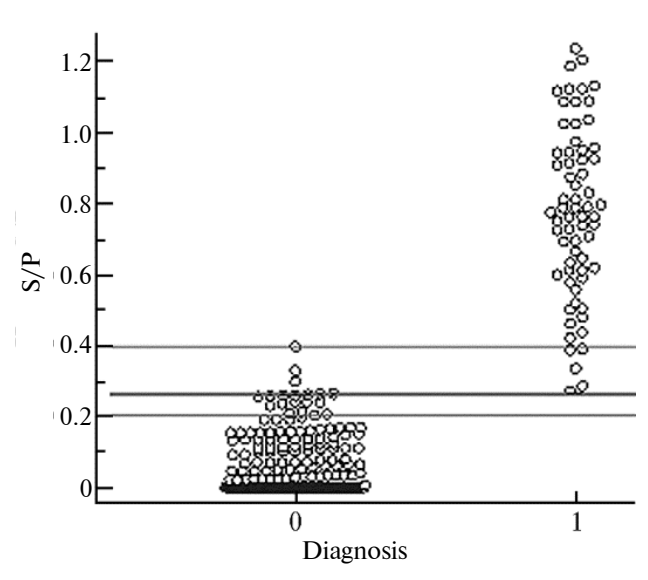

Fig. 1. Distribution of 410 reference negative ( $\mathrm{Di}-$ agnosis 0) and 66 reference positive for African swine fever (Diagnosis 1) samples of pig blood serum in indirect ELISA by $\mathbf{S} / \mathbf{P}$ index (the ratio of the test sample to positive control optical density). Each point represents the average of individual sample analysis duplicates. A sensitivity of $100 \%$ and a specificity of $99.3 \%$ correspond to the cutoff line at $\mathrm{S} / \mathrm{P}>0.264$. The $\mathrm{S} / \mathrm{P}$ area boundaries are marked in which both positive and negative samples are co-located.

Sensitivity and specificity of indirect ELISA blood test were calculated for different cutoff values (Table 2). In the samples from domestic pigs and wild boars, the highest diagnostic sensitivity $(100 \%)$ at $95 \%$ con-

fidence interval (CI of 94.6 to $100 \%$ ) along with the highest specificity of $99.27 \%$ (95\% CI: 97.9-99.8\%) was observed at the cutoff value of 0.264 .

2. Criteria and ROC curve coordinates for indirect ELISA tests of serum antiASFV antibodies depending on the cutoff value

\begin{tabular}{ccccccc|c|c|c}
\hline Criterion & Sen, \% & 95 \% CI & Sp, \% & 95 \% CI & +LR & 95 \% CI & -LR & 95 \% CI \\
\hline$>0.258$ & 100.00 & $94.6-100.0$ & 98.54 & $96.8-99.5$ & 68.33 & $30.9-151.2$ & 0.000 & \\
$>0.261$ & 100.00 & $94.6-100.0$ & 98.78 & $97.2-99.6$ & 82.00 & $34.3-196.0$ & 0.000 & \\
$>0.263$ & 100.00 & $94.6-100.0$ & 99.02 & $97.5-99.7$ & 102.50 & $38,7-271,8$ & 0.000 & \\
$>0.264$ & 100.00 & $94.6-100.0$ & 99.27 & $97.9-99.8$ & 136.67 & $44.3-422.0$ & 0.000 & \\
$>0.271$ & 98.48 & $91.8-100.0$ & 99.27 & $97.9-99.8$ & 134.60 & $43.6-415.8$ & 0.015 & $0.002-0.1$ \\
$>0.285$ & 96.97 & $89.5-99.6$ & 99.27 & $97.9-99.8$ & 132.53 & $42.9-409.5$ & 0.031 & $0.008-0.1$ \\
$>0.298$ & 96.97 & $89.5-99.6$ & 99.51 & $98.2-99.9$ & 198.79 & $49.9-792.7$ & 0.030 & $0.008-0.1$ \\
$>0.388$ & 93.94 & $85,2-98,3$ & 99.76 & $98.6-100.0$ & 385.15 & $54.3-2730.4$ & 0.061 & $0.020-0.2$
\end{tabular}

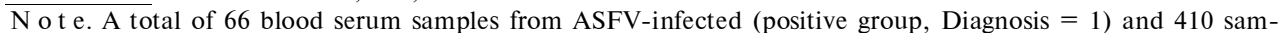
ples from non-infected pigs and wild boars (negative group, Diagnosis $=0$ ) have been tested; 476 samples in total). Sen - sensitivity, Sp - specificity, CI - confidence interval, +LR and -LR mean the likelihood ratio: $+\mathrm{LR}=\mathrm{Sen} /(100-\mathrm{Sp}),-\mathrm{LR}=(100-\mathrm{Sen}) / \mathrm{Sp}$. The table shows part of ROC analysis criteria and coordinates calculated in the MedCalc program for the most relevant cutoff values in the range of optimal sensitivity and specificity. Area under the ROC curve (AUC): area under the ROC curve -1.000 ; standard error -0.000269 ; $95 \%$ confidence interval - from 0.992 to 1.000 ; Z-statistics -1857.961 ; significance level $\mathrm{P}($ area $=0.5)<0.0001$. Youden index: index $\mathrm{J}-0.9927$; associative criterion $>0.264$.

When testing 59 reference positive and 678 reference negative spleen tissue extracts (Fig. 2), the S/P overlap frequencies of false positive (51 samples) and false-negative (3 samples) results were in the same range (0.2-0.4) as for the serum antibodies. The optimal parameters were at a cutoff value of 0.284 : method sensitivity was $100 \%$ (95\% CI: 93.9-100\%), specificity was $98.82 \%(95 \%$ CI: 97.7-99.5\%) (Table 3).

Currently, ELISA is approved by OIE for primary screening anti-ASFV antibodies [26], but in general all of the known tests allow detecting specific antibodies in blood only [10, 12, 14]. The validation of ELISA kit by ROC analysis showed the possibility to use this kit for the detection of specific anti-ASFV antibodies in blood and tissue extracts in infected domestic pigs and wild boars. Examination of positive $(n=66)$ and negative $(n=410)$ pig blood samples confirmed high sensitivity and specificity of the method compared to IIFT. Only 8 $(1.6 \%)$ of the 476 samples were within the positive/negative cutoff area (see Fig. 1). 


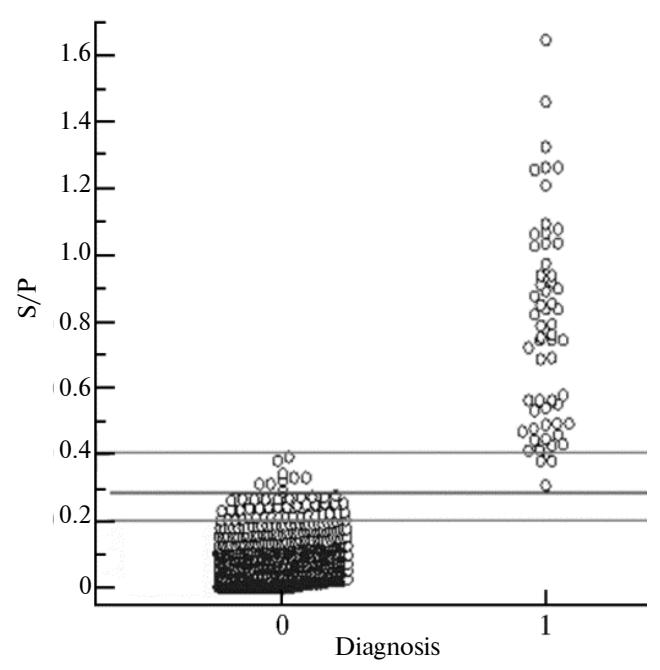

Considering the acute disease character, we can assume a state corresponding to the incubation period of infection in the animals with positive reactions.

3. Criteria and ROC curve coordinates for indirect ELISA tests of serum antiASFV antibodies in spleen tissue depending on the cutoff value

\begin{tabular}{c|c|c|c|c|c|c|c|c}
\hline Criterion & Sen, \% & $95 \%$ CI & Sp, \% & $95 \%$ CI & +LR & $95 \%$ CI & -LR & $95 \%$ CI \\
\hline$>0.263$ & 100.00 & $93.9-100.0$ & 97.94 & $96.6-98.9$ & 48.43 & $28.8-81.3$ & 0.000 & \\
$>0.268$ & 100.00 & $93.9-100.0$ & 98.08 & $96,7-99,0$ & 52.15 & $30.4-89.4$ & 0.000 & \\
$>0.269$ & 100.00 & $93.9-100.0$ & 98.23 & $96.9-99.1$ & 56.50 & $32.2-99.0$ & 0.000 & \\
$>0.270$ & 100.00 & $93.9-100.0$ & 98.53 & $97.3-99.3$ & 67.80 & $36.6-125.4$ & 0.000 & \\
$>0.276$ & 100.00 & $93.9-100.0$ & 98.67 & $97.5-99.4$ & 75.33 & $39.4-144.2$ & 0.000 & \\
$>0.284$ & 100.00 & $93.9-100.0$ & 98.82 & $97.7-99.5$ & 84.75 & $42.6-168.8$ & 0.000 & \\
$>0.304$ & 98.31 & $90.9-100.0$ & 98.82 & $97.7-99.5$ & 83.31 & $41.8-166.1$ & 0.017 & $0.002-0.1$ \\
$>0.310$ & 98.31 & $90.9-100.0$ & 99.12 & $98.1-99.7$ & 111.08 & $50.0-246.6$ & 0.017 & $0.002-0.1$ \\
$>0.320$ & 98.31 & $90.9-100.0$ & 99.26 & $98.3-99.8$ & 133.30 & $55.6-319.4$ & 0.017 & $0.002-0.1$ \\
$>0.330$ & 98.31 & $90.9-100.0$ & 99.56 & $98.7-99.9$ & 222.17 & $71.8-687.5$ & 0.017 & $0.002-0.1$ \\
$>0.380$ & 94.92 & $85.9-98.9$ & 99.85 & $99.2-100.0$ & 643.53 & $90.7-4566.1$ & 0.051 & $0.020-0.2$
\end{tabular}

Note. A total of 59 spleen samples from ASFV-infected (positive group, Diagnosis $=1$ ) and 678 samples from non-infected pigs and wild boars (negative group, Diagnosis $=0$ ) have been tested; 737 samples in total. Sen - sensitivity, Sp - specificity, CI - confidence interval, +LR and - LR mean the likelihood ratio: $+\mathrm{LR}=\mathrm{Sen} /(100-\mathrm{Sp}),-\mathrm{LR}=(100-\mathrm{Sen}) / \mathrm{Sp}$. The table shows part of ROC analysis criteria and coordinates calculated in the MedCalc program for the most relevant cutoff values in the range of optimal sensitivity and specificity. Area under the ROC curve (AUC): area under the ROC curve -1.000 ; standard error -0.000243 ; $95 \%$ confidence interval - from 0.994 to 1.000 ; Z-statistics -2055.362 ; significance level P $($ area $=0.5)<0.0001$. Youden index: index $\mathbf{J}-0.9882$; associative criterion $>0.284$.

The results of indirect ELISA ROC analysis obtained in the study of blood and tissue samples allow choosing cutoff points in a wide range with the possibility of optimizing method performance. In both cases, alternative options are possible depending on the planned purposes, such as diagnosis, monitoring, or confirmation of the absence of ASF infection in the population of domestic pigs (farm) or wild boars (hunting facilities). Thus, if it is necessary to prevent the introduction of infection, high sensitivity is more important and specificity is of less significance (e.g. in diagnosis or screening in an affected area). In this case, the set cutoff level is 0.264 (blood) or 0.284 (tissue). If a false positive result is inadmissible, that is, specificity is important (e.g. when screening animals for ASF in an ASF-free area), the cutoff threshold can be increased to 0.380 with a minimal effect on sensitivity.

Thus, the validation indicates that the VNIIVViM ASF-ELISA Ab/Ag kit allows reliable detection of anti-ASFV antibodies both in the blood serum and spleen tissue extracts at S/P cutoff values of 0.264 (blood) or 0.284 (tissue), re- 
spectively. This is especially important for monitoring wild boars after shooting, when only organs are often delivered to the laboratory. The commercial set developed and validated by us is expedient for using to evaluate the presence of antiASFV antibodies and the ASF status of an animal and the population as a whole.

\section{REFERE N C ES}

1. Kurinnov V.V., Kolbasov D.V., T Sybanov S.Zh., Vasil'ev A.P., Shendrik A.G., B alyshev V.M., Mikolaichuk S.V., B elyan in S.A., Kalantaenko Yu.F., Z h u k o v A.N. Zhizn' bez opasnostei, 2010, 3: 82-87 (in Russ.).

2. Atuhaire D.K., Afayoa M., Ochwo S., Mwesigwa S., Mwiine F.N., Okuni J.B., O l a ho-Mukani W., O jok L. Prevalence of African swine fever virus in apparently healthy domestic pigs in Uganda. BMC Vet. Res., 2013, 9: 263-271 (doi: 10.1186/1746-6148-9-263).

3. Gallardo C., Reoyo A.T., Fernández-Pinero J., Iglesias I., Mucoz J., Arias L. African swine fever: a global view of the current challenge. Porcine Health Management, 2015, 1: 21 (doi: 10.1186/s40813-015-0013-y).

4. Carras co L. An update on the epidemiology and pathology of African swine fever. J. Comp. Pathol., 2015, 152: 9-21 (doi: 10.1016/j.jcpa.2014.09.003).

5. Guinat C., Reis A.L., Netherton C., Goatley L., Pfeiffer D., Dixon L. Dynamics of African swine fever virus shedding and excretion in domestic pigs infected by intramuscular inoculation and contact transmission. Vet. Res., 2014, 45: 93 (doi: 10.1186/s13567-014-0093-8).

6. Diseases of swine. D.J. Taylor, J.J. Zimmerman, S. D’Allaire (eds.) Iowa State University Press, 2006.

7. Gallardo C., Soler A., Nieto R., Cano C., Pelayo V., Sánchez M.A., Pridotkas G., Fernandez-Pinero J., Briones V., Arias M. Experimental infection of domestic pigs with African swine fever virus Lithuania 2014 genotype II field isolate. Transbound. Emerg. Dis., 2015 Mar 22: 1-5 (doi: 10.1111/tbed.12346) [Epub ahead of print].

8. Boinas F.S., Hutchings G.H., Dix on L.K., Wilkins on P.J. Characterisation of pathogenic and non-pathogenic African swine fever virus isolated from Ornithodoros erraticus inhabiting pig premises in Portugal. J. Gen. Virol., 2004, 5(8): 2117-2187 (doi: 10.1099/vir.0.80058-0).

9. Howey E.B., O'Donnell V., de Carvalho Ferreira H.C., Borca M.V., Arzt J. Pathogenesis of highly virulent African swine fever virus in domestic pigs exposed via intraoropharyngeal, intranasopharyngeal, and intramuscular inoculation, and by direct contact with infected pigs. Virus Res., 2013, 178: 328-339 (doi: 10.1016/j.virusres.2013.09.024).

10. Charleston B., Bankowski B.M., Gubbins S., Chase-Topping M.E., Schley D., Howey R., B arnett P.V., Gibson D., Jule ff N.D., Woolhouse M.E. Relationship between clinical signs and transmission of an infectious disease and the implications for control. Science, 2011, 332: 726-729 (doi: 10.1126/science.1199884).

11. B otija S., O rdas A. Rapid diagnosis by identification of antibodies extracted from tissues using indirect immunofluorescence. Hog Cholera. In: Classical swine fever and African swine fever. Luxembourg, 1977: 658-659.

12. Re is A.L., Parkhouse R.M., Pe nedos A.R., Martins C., Le it ro A.. Systematic analysis of longitudinal serological responses of pigs infected experimentally with African swine fever virus. J. Gen. Virol., 2007, 88: 2426-2434 (doi: 10.1099/vir.0.82857-0).

13. Pérez J., Fernández A.I., Sierra M.A., Herraez P., Fernández A. Serological and immunohistochemical study of African swine fever in wild boar in Spain. Vet Rec., 1998, 143: 136-139.

14. Gallardo C., Soler A., Nieto R., Carrascosa A.L., De Mia G.M., B ishop R.P., Martins C., F asina F.O., Cou acy-H y m an E., He at h L., Pela yo V., Martín E., S i my n A., Martín R., Okurut A.R., Lekolol I., Okoth E., Arias M. Comparative evaluation of novel African swine fever virus (ASF) antibody detection techniques derived from specific ASF viral genotypes with the OIE internationally prescribed serological tests. Vet. Microbiol., 2013, 162: 32-43 (doi: 10.1016/j.vetmic.2012.08.011).

15. Kurinnov V.V., Vasil'ev A.P., Belyanin S.A., Strizhakova O.M., Nogina I.V., Sidlik M.V., Gazaev I.Kh., Tsybanov S.Zh., Mironova L.P., Alikova G.A., Dzhailidi G.A., Chernykh O.Yu. Veterinariya Kubani, 2014, 3: 5-9 (in Russ.).

16. Kurinnov V.V., Belyanin S.A., Vasil'ev A.P., Strizhakova O.M., Lyska V.M., Nogina I.V., Zubairova S.N., Balyshev V.M., Tsybanov S.Zh., Kolbasov D.V., M ironova P.L., Che rnykh O.Yu., Alikova G.A. Veterinariya Kubani, 2012, 4: 9-11 (in Russ.).

17. Cubillos C., Gymez-Sebastian S., Moreno N., Nuñez M.C., MulumbaM fu mu L.K., Que mbo C.J., He ath L., Ette r E.M.C., Jori F., Escribano J.M., $\mathrm{B} 1 \mathrm{a} \mathrm{n} \mathrm{co}$ E. African swine fever virus serodiagnosis: A general review with a focus on the analyses of African serum samples. Virus Res., 2013, 173(1): 159-167 (doi: 10.1016/j.virusres.2012.10.021).

18. Nieto-Pelegr E., Rivera-Arroyo B., S a nchez-Vizca 1 no J.M. First detection of antibodies against African swine fever virus in faeces samples. Transbound. Emerg. Dis., 2015, 
62(6): 594-602 (doi: 10.1111/tbed.12429).

19. Zsak L., Borca M., Risatti G., Zsak A., French R., Lu Z., Kutish G., N e ilan J., Callahan J., Nels on W. Preclinical diagnosis of African swine fever in contact-exposed swine by a real-time PCR assay. J. Clin. Microbiol., 2005, 43: 112-119 (doi: 10.1128/JCM.43.1.112-119.2005).

20. Makarov V.V., Sukharev O.I., Boev B.V., Gavryushkin D., Kolomytsev A.A., Li t vi nov O.B. Veterinariya, 2010, 9: 24-28 (in Russ.).

21. Khomenko S., Beltrán-Alcrudo D., Rozstalnyy A., Gogin A., Kolbasov D., Pinto J., Lubroth J., Martin V. African swine fever in the Russian Federation: risk factors for Europe and beyond. EMPRES Watch, 2013, 28: 1-14.

22. Manual of diagnostic tests and vaccines for terrestrial animals. $7^{\text {th }}$ Ed. OIE, France. 2012. Available http://www.oie.int/manual-of-diagnostic-tests-and-vaccines-for-terrestrial-animals/. No date.

23. Gardner A., Greiner M., Dubey J. Statistical evaluation of test accuracy studies for Toxoplasma gondii in food animal intermediate hosts. Zoonoses and Public Health, 2010, 57: 8294 (doi: 10.1111/j.1863-2378.2009.01281).

24. Greiner M., Sohr D., Gijbel P. A modified ROC analysis for the selection of cut-off values and the definition of intermediate results of serodiagnostic tests. J. Immunol. Methods, 1995, 185: $123-132$.

25. The ELISA guidebook. J.R. Crowther (ed.). Vienna, 2009.

26. J a c obson R.H. Validation of serological assays for diagnosis of infectious diseases. Rev. Sci. Tech. OIE, 1998, 17(2): 469-486.

27. Tignon M., Gallardo C., Iscaro C., Hutet E., Van der Stede Y., Kolbasov D., De Mia G.M., Le Potier M.-F., Bishop R.P., Arias M. Development and interlaboratory validation study of an improved new real-time PCR assay with internal control for detection and laboratory diagnosis of African swine fever virus. J. Virol. Methods, 2011, 178: 161-170 (doi: 10.1016/j.jviromet.2011.09.007). 